'Escuela de Salud Pública, Facultad de Medicina, Universidad de Chile. Santiago, Chile.

2Escuela de Enfermería, Facultad de Salud, Universidad Bernardo

O`Higgins. Santiago, Chile.

${ }^{3}$ Programa Doctorado en Salud

Pública. Escuela de Salud

Pública. Facultad de Medicina,

Universidad de Chile. Santiago,

Chile.

${ }^{a}$ Enfermera, Magíster en Bioética. ${ }^{\mathrm{b}}$ Enfermera Matrona. 'MPH.

dPhD.

Los autores declaran no tener conflicto de interés. Trabajo no recibió financiamiento.

Recibido el 4 de enero de 2018 , aceptado el 5 abril de 2019 .

Correspondencia: Carolina Nazzal Nazzal Independencia 939. Santiago, Chile. cnazzal@med.uchile.cl

\section{Tendencia de las defunciones ocurridas en mayores de 1 año según lugar de ocurrencia y su relación con características sociodemográficas, Chile 1997-2014}

\author{
MARÍA CRISTINA PAREDES E. 2,3,a, ALONSO FAUSTINO ${ }^{1,3, c,}$ \\ CAROLINA NAZZAL N. ${ }^{1, b, c, d}$
}

\section{Temporal variation in the place of death in Chile from 1997 to 2014}

Background: The place of death is a fundamental indicator for the debate on equity and access to health care. Aim: To describe the place of death of the deceased population over 1 year of age in Chile between the years 1997 and 2014. To analyze tendencies in this variable and its association with socio-demographic characteristics. Material and Methods: Time series study covering deaths occurred between 1997 and 2014 in Chile. National death records were used, provided by the Department of Health Statistics and Information (DEIS) of the Chilean Ministry of Health. The following variables were chosen: place of death (home, hospital, other), sex, marital status, age, level of education, activity and area of residence. Temporal trends were evaluated using Prais Winsten regressions. Logistic regression was used to assess the association of the place of death with socio-demographic characteristics. Results: Between 1997 and 2014 there were 1,576,392 deaths, at a mean age of 69 years ( $p 25-p 75: 60-83$ years). No temporal variations in the place of death were observed with the Prais Winsten regression, hospital ( $P$-W coefficient (coef) $=0.06$ (confidence intervals $(C I)$ : $-0.30 ; 0.19)$, $p=0.64)$, home $(P-W$ coef $=-0.03(C I:-0.15 ; 0.09), p=0.57)$, and other places $(P-W$ coef $=0.07 ;(C I:-0.08-0.22), p=0.32)$. The multivariate analysis showed that being women under 70 years of age, being married or widowed, having a higher educational level, being inactive and living in a rural area were factors associated with a greater chance of dying at home. Conclusions: No significant temporal variation in the place of death was observed.

(Rev Med Chile 2019; 147: 322-329)

Key words: Cause of Death; Chile; Death; Mortality.
L a mortalidad constituye un parámetro fundamental que informa sobre los cambios demográficos que ocurren en la población y su análisis ha contribuido al desarrollo de políticas públicas con enfoques de prevención y tratamiento. En el año 1950, la esperanza de vida en Chile era 54,8 años; para el quinquenio 2020-2025 se espera que sea 80,2 años ${ }^{1}$, lo que refleja el éxito e impacto de las estrategias implementadas en el aumento considerable de este indicador. El avance de la tecnología biomédica y la medicalización representan factores relacionados con el aumento de la esperanza de vida. Si bien esto implica una vida más larga, las consecuencias a nivel social 
y económico no dejan de ser relevantes para el sistema de salud ${ }^{2}$. Es así que para el año 2050 se proyecta que $22 \%$ de la población mundial pertenecerá al grupo etario de personas mayores de 65 años; en el caso de Chile se estima que será $21,6 \%$, lo que se traducirá en aumento del número de defunciones ${ }^{3-5}$.

La defunción, como proceso, muestra cambios históricos en su desarrollo y significado. El paso de la muerte en domicilio a la muerte hospitalaria a partir de la mitad del siglo XIX generó una cierta intolerancia frente a la muerte $e^{6,7}$. En el hospital, la muerte se desencadenaba como un fenómeno técnico, resultado del cese de los cuidados, desembocando en un modelo de muerte hospitalaria, es decir, muerte medicalizada ${ }^{8}$. Este fenómeno es una característica de las sociedades actuales, en la que se ha transferido a la ciencia, y en particular a la medicina, la responsabilidad de enfrentar el proceso $^{7,8}$, generando desafíos e incertidumbres para pacientes, familiares y profesionales.

Se ha visto que la edad es un determinante del lugar de ocurrencia de la muerte; en la medida que aumenta la edad, también aumenta la probabilidad de morir en la casa ${ }^{9}$. En Chile existen datos que revelan este mismo fenómeno, así como una tendencia hacia la ocurrencia de las defunciones en el hospital según la causa de muerte ${ }^{10}$.

El objetivo de este estudio fue describir el lugar de ocurrencia de las defunciones en Chile entre los años 1997 y 2014 en población mayor de 1 año y analizar su tendencia, así como su asociación con la edad, sexo, nivel educacional, condición laboral, estado civil, ruralidad y regiones del país. Para ello se emplearon los registros de estadísticas vitales que permiten analizar la ocurrencia de las muertes en el tiempo ${ }^{11}$.

\section{Material y Método}

\section{Diseño y población}

Estudio de series de tiempo; se incluyeron todas las defunciones ocurridas en mayores de 1 año en Chile entre 1997 y 2014 (se excluyeron los menores de un año, ya que la mayoría de la mortalidad infantil ocurre en ambiente hospitalario).

Recolección de información: se emplearon las bases de datos de defunciones de Chile ocurridas entre los años 1997 y 2014, disponibles en el sitio web del Departamento de Estadísticas e Información en Salud (DEIS) del Ministerio de Salud de
Chile ${ }^{1}$. Para cada defunción se obtuvo información sobre lugar de ocurrencia de la defunción, edad en años cumplidos, sexo, estado civil, nivel educacional, actividad, atención médica, región y mes de defunción.

El lugar de ocurrencia de la defunción se evaluó como variable respuesta, de acuerdo al esquema de registro que las agrupa según si ocurrieron en hospital o clínica, casa o domicilio y otro. La actividad consigna si la persona se encontraba activa o inactiva al momento de la defunción en términos de ocupación laboral. La variable región considera la división político-administrativa, incluyendo las 15 regiones actuales. El estado civil se categorizó como soltero, casado o viudo, incluyendo a los divorciados en la categoría soltero. El nivel educacional se agrupó según el nivel máximo de estudio completado en ninguno, básico, medio o superior.

\section{Análisis estadístico}

Se identificaron duplicados y valores perdidos o inconsistentes, los que fueron tratados como datos faltantes; estos fueron menos del $1 \%$ de los datos. Las variables categóricas fueron descritas a través de frecuencias absolutas y relativas, comparándose a través del uso del test estadístico de $\chi^{2}$. Las variables continuas fueron descritas con medianas y percentiles. Para el análisis de tendencia se calcularon las proporciones de defunciones según lugar de ocurrencia para cada año de defunción. La tendencia se evaluó mediante un modelo de regresión de Prais-Winsten; este modelo, similar a una regresión lineal, se emplea cuando existe dependencia temporal de las observaciones (autocorrelación) y entrega un coeficiente indicando el cambio anual en el estimador calculado ${ }^{12}$. Para el estudio de la relación entre las características sociodemográficas del fallecido y el lugar de ocurrencia de la defunción se calcularon odds ratios (OR) a través de modelos de regresión logística crudo. Se evaluaron interacciones, identificando a la edad como una variable modificadora de efecto, por lo cual se realizó un análisis multivariado estratificado por edad en dos grupos, menores de 70 y mayor o igual a 70 años (ajustado por sexo, estado civil, nivel educacional, actividad y ruralidad). El nivel de significación considerado fue 5\% y se utilizó el software estadístico STATA 13.

Este estudio no requirió evaluación de Comité de Ética por tratarse de bases secundarias cuyos datos son anónimos. 


\section{Resultados}

Entre los años 1997 y 2014 ocurrieron 1.576.306 defunciones en mayores de 1 año. En hospital o clínica se registraron 697.044 (44,2\%) defunciones, en casa o domicilio 728.190 (46,2\%) y en otro lugar $151.072(9,6 \%)$.

La mediana de edad fue 74 años $\left(\mathrm{P}_{25}-\mathrm{P}_{75}: 60\right.$ $83)$; en hombres fue 70 años $\left(\mathrm{P}_{25}-\mathrm{P}_{75}: 56-80\right)$ y en mujeres 78 años $\left(\mathrm{P}_{25}-\mathrm{P}_{75}: 65-86\right)$. La proporción de defunciones en personas de 70 o más años fue $59,2 \%$ (932.963). Las defunciones ocurrieron con mayor frecuencia en los meses de junio, julio y agosto, acumulando estos tres meses $28,3 \%$ del total de defunciones.

Las características sociodemográficas de las defunciones según lugar de ocurrencia se muestran en la Tabla 1. Los hombres presentaron significativamente más muertes en el hospital o clínica, así como en la categoría "otro lugar de defunción", en comparación con las mujeres $(\mathrm{p}<0,001)$. Situación similar ocurrió con el grupo de personas solteras, en comparación con las casadas y viudas $(\mathrm{p}<0,001)$. En relación al nivel educacional, las personas que presentan nivel educacional básico, comparadas con aquellas de nivel medio y alto fallecieron más en casa o habitación $(\mathrm{p}<0,001)$. Al momento de la defunción, $85,1 \%$ (1.341.103) se encontraba inactivo laboralmente, siendo esta categoría aun más frecuente en los fallecidos en casa.

La Figura 1 muestra la proporción de defunciones según lugar de ocurrencia por región. En las regiones de Coquimbo (4), Araucanía (9), Los Lagos (10), Los Ríos (14) y Arica y Parinacota (15) las defunciones se presentaron con mayor proporción en casa o habitación.

Del total de las defunciones ocurridas en el período según lugar de ocurrencia y decenios de

Tabla 1. Características socio-demográficas según lugar de ocurrencia de la defunción en mayores de 1 año, Chile 1997-2014

\begin{tabular}{|c|c|c|c|c|}
\hline Lugar de la defunción & $\begin{array}{l}\text { Hospital o clínica } \\
697.044(44,2 \%)\end{array}$ & $\begin{array}{c}\text { Casa o habitación } \\
728.190(46,2 \%)\end{array}$ & $\begin{array}{c}\text { Otro lugar } \\
151.072(9,6 \%)\end{array}$ & Total \\
\hline \multicolumn{5}{|l|}{ Sexo* } \\
\hline Hombres & $381.622(45,0)$ & $358.340(42,2)$ & $108.317(12,8)$ & $848.279(100)$ \\
\hline Mujeres & $315.422(43,3)$ & $369.850(50,8)$ & $42.755 \quad(5,9)$ & $728.027(100)$ \\
\hline \multicolumn{5}{|l|}{ Estado civil* } \\
\hline Soltero & $245.171(44,4)$ & $229.558(41,5)$ & $77.829(14,1)$ & $552.558(100)$ \\
\hline Casado & $388.355(44,6)$ & $417.346(47,9)$ & $64.939(7,5)$ & $870.640(100)$ \\
\hline Viudo & $61.357(41,4)$ & $79.516(53,6)$ & $7.526 \quad(5,0)$ & $148.709(100)$ \\
\hline Ignorado & $2.161(45,9)$ & $1.770(37,6)$ & $778(16,5)$ & $4.709(100)$ \\
\hline \multicolumn{5}{|l|}{ Nivel educacional* } \\
\hline Superior & $31.271(44,2)$ & $30.001(42,4)$ & $9.537(13,4)$ & $70.809(100)$ \\
\hline Media & $192.480(44,8)$ & $186.687(43,5)$ & $50.457(11,7)$ & $429.624(100)$ \\
\hline Básica & $376.923(43,6)$ & $413.039(47,8)$ & $74.792 \quad(8,6)$ & $864.754(100)$ \\
\hline Ninguno & $95.660(45,7)$ & $97.939(46,8)$ & $15.753(7,5)$ & $209.352(100)$ \\
\hline Ignorado & $690(39,2)$ & $524(29,6)$ & $553(31,2)$ & $1.767(100)$ \\
\hline \multicolumn{5}{|l|}{ Actividad laboral* } \\
\hline Inactivo & $594.136(44,3)$ & $655.764(48,9)$ & $91.203 \quad(6,8)$ & $1.341 .103(100)$ \\
\hline Activo & $102.120(43,8)$ & $71.841(30,8)$ & $59.122(25,8)$ & $233.083(100)$ \\
\hline Ignorado & $788(37,2)$ & $585(27,6)$ & $747(35,2)$ & $2.120(100)$ \\
\hline \multicolumn{5}{|l|}{ Área* } \\
\hline Urbana & $605.947(44,9)$ & $620.787(46,0)$ & $122.958 \quad(9,1)$ & $1.349 .136(100)$ \\
\hline Rural & $91.097(40,2)$ & $107.403(47,4)$ & $28.114(12,4)$ & $226.614(100)$ \\
\hline
\end{tabular}

${ }^{*} \mathrm{p}<0,05$ para todas las comparaciones, mediante el test estadístico de $\chi^{2}$, entre las distintas categorías de lugar de defunción. 


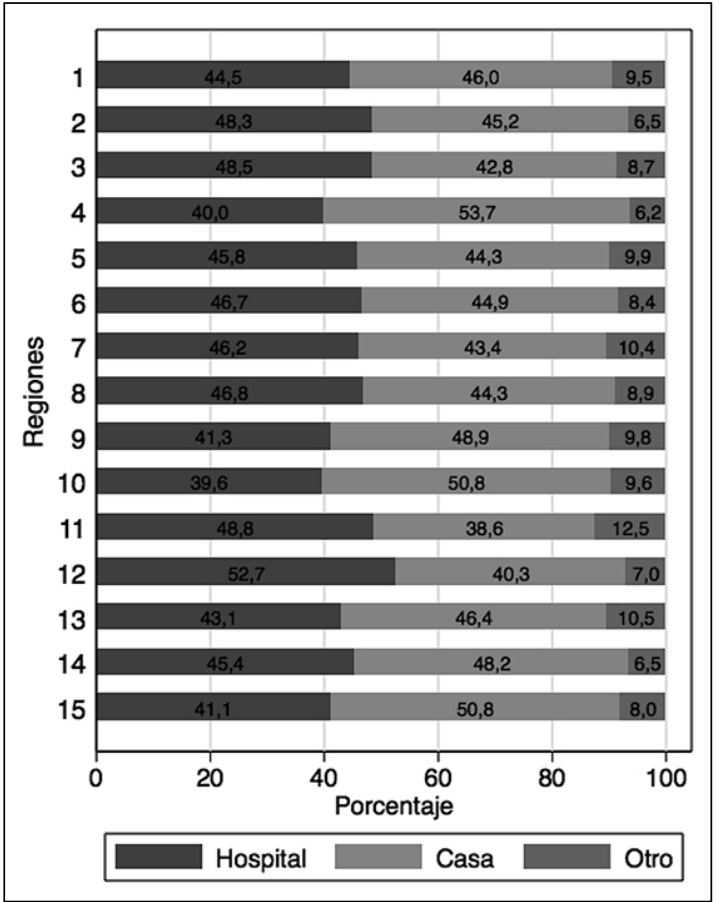

Figura 1. Proporción de las defunciones según lugar de ocurrencia y regiones, Chile 1997-2014. edad, la mayoría de ellas ocurrió en el hospital, excepto en los mayores de 70 años, donde el lugar más frecuente fue la casa o habitación (Figura 2).

El análisis temporal no mostró variaciones ni una tendencia significativa para el lugar de defunción (Figura 3). Los resultados de la regresión de Prais Winsten según lugar de ocurrencia fueron: para muertes ocurridas en el hospital, coef $\mathrm{P}-\mathrm{W}=-0,06,95 \%$ IC $[-0,30,0,19], \mathrm{p}=0,64$, en domicilio, coef $\mathrm{P}-\mathrm{W}=-0,03,95 \%$ IC $[-0,15,0,09]$, $\mathrm{p}=0,57$, y en otro lugar, coef $\mathrm{P}-\mathrm{W}=0,07,95 \%$ IC $[-0,08,0,22], \mathrm{p}=0,32$.

El análisis de OR crudo mostró que por cada año que aumenta la edad, se incrementa el riesgo de fallecer en casa en 2,4\%. El análisis de OR multivariado mostró para los menores de 70 años de sexo femenino, estado civil casado o viudo, mayor nivel educacional, inactividad y área rural se asociaron con mayor probabilidad de morir en el domicilio. En los mayores de 70 años se encontraron los mismos factores de riesgo, a excepción de estado civil casado y mayor nivel educacional, los que se asociaron con menor probabilidad de morir en el domicilio (Tabla 2).

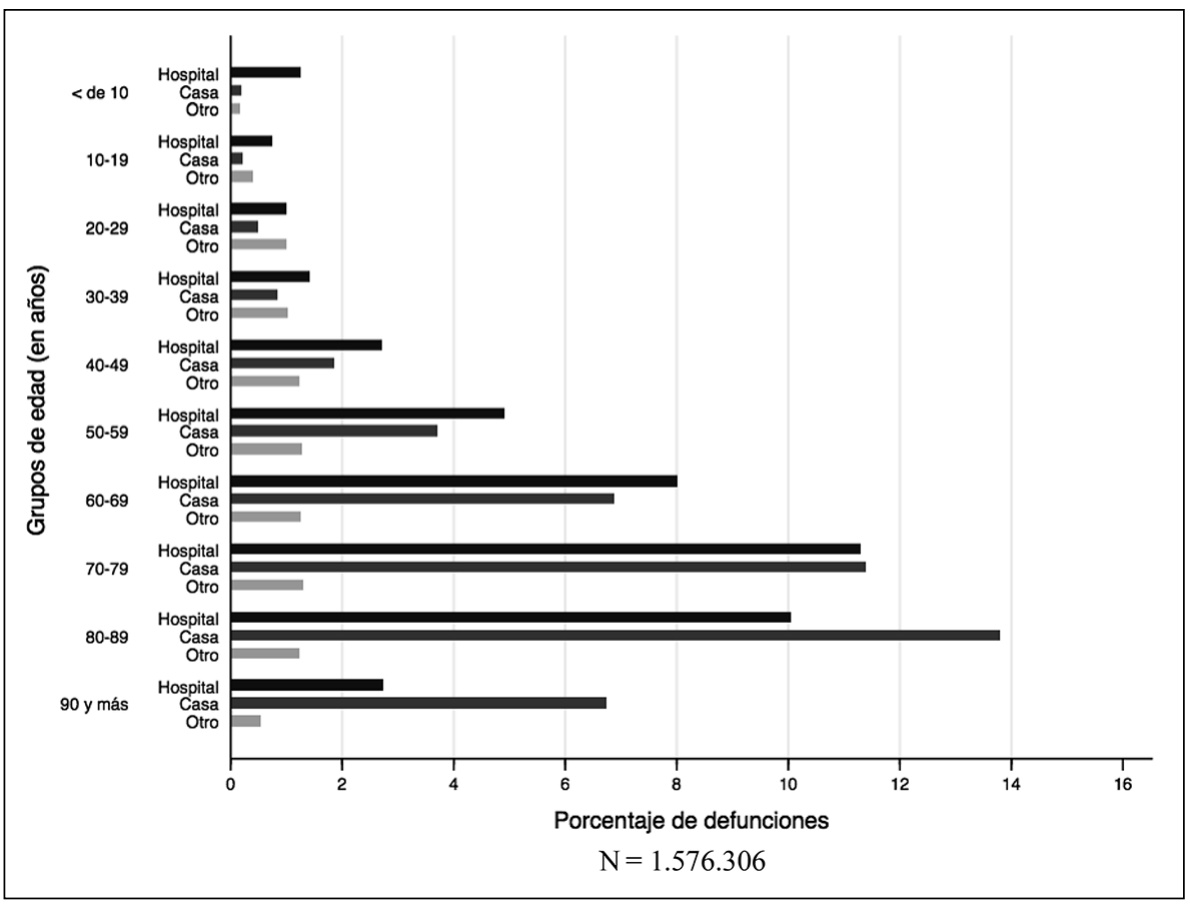

Figura 2. Defunciones en mayores de 1 años, según lugar de ocurrencia y decenios de edad, Chile 1997 y 2014. 
Tabla 2. OR y 95\% IC de factores sociodemográficos asociados a muerte en el domicilio versus muerte en hospital para las defunciones en mayores de 1 año. Chile, 1997-2014

\begin{tabular}{|c|c|c|c|c|c|c|}
\hline & \multicolumn{2}{|c|}{ Crudo } & \multicolumn{2}{|c|}{$\begin{array}{l}\text { Multivariado para } \\
\text { menores de } 70 \text { años }\end{array}$} & \multicolumn{2}{|c|}{$\begin{array}{l}\text { Multivariado para } \\
\text { mayores de } 70 \text { años }\end{array}$} \\
\hline & OR & $95 \%$ IC & OR & $95 \%$ IC & OR & $95 \%$ IC \\
\hline \multicolumn{7}{|l|}{ Sexo } \\
\hline Masculino & 1 & - & 1 & - & 1 & - \\
\hline Femenino & 1,249 & $(1,241-1,257)$ & 1,088 & $(1,075-1,101)$ & 1,174 & $(1,164-1,184)$ \\
\hline \multicolumn{7}{|l|}{ Estado civil casado } \\
\hline Soltero & 1 & - & 1 & - & 1 & - \\
\hline Casado & 1,148 & $(1,140-1,156)$ & 1,161 & $(1,148-1,175)$ & 0,982 & $(0,973-0,992)$ \\
\hline Viudo & 1,384 & $(1,368-1,401)$ & 1,183 & $(1,150-1,217)$ & 1,037 & $(1,022-1,052)$ \\
\hline \multicolumn{7}{|l|}{ Nivel educacional } \\
\hline Ninguno & 1 & - & 1 & - & 1 & - \\
\hline Nivel educacional básico & 1,070 & $(1,060-1,081)$ & 1,630 & $(1,582-1,680)$ & 0,885 & $(0,874-0,897)$ \\
\hline Nivel educacional media & 0,947 & $(0,937-0,958)$ & 1,659 & $(1,626-1,694)$ & 0,870 & $(0,858-0,883)$ \\
\hline Nivel educacional superior & 0,937 & $(0,920-0,954)$ & 1,722 & $(1,689-1,755)$ & 0,970 & $(0,945-0,995)$ \\
\hline \multicolumn{7}{|l|}{ Activo al momento de la defunción } \\
\hline Activo & 1 & - & 1 & - & 1 & - \\
\hline Inactivo & 1,569 & $(1,553-1,585)$ & 1,128 & $(1,113-1,143)$ & 1,048 & $(1,016-1,082)$ \\
\hline \multicolumn{7}{|l|}{ Área de la defunción } \\
\hline Urbano & 1 & - & 1 & - & 1 & - \\
\hline Rural & 1,151 & $(1,140-1,162)$ & 1,312 & $(1,291-1,333)$ & 1,090 & $(1,076-1,104)$ \\
\hline Edad al momento de la defunción & 1,024 & $(1,023-1,024)$ & - & - & - & - \\
\hline
\end{tabular}

Referencia: Hospital.

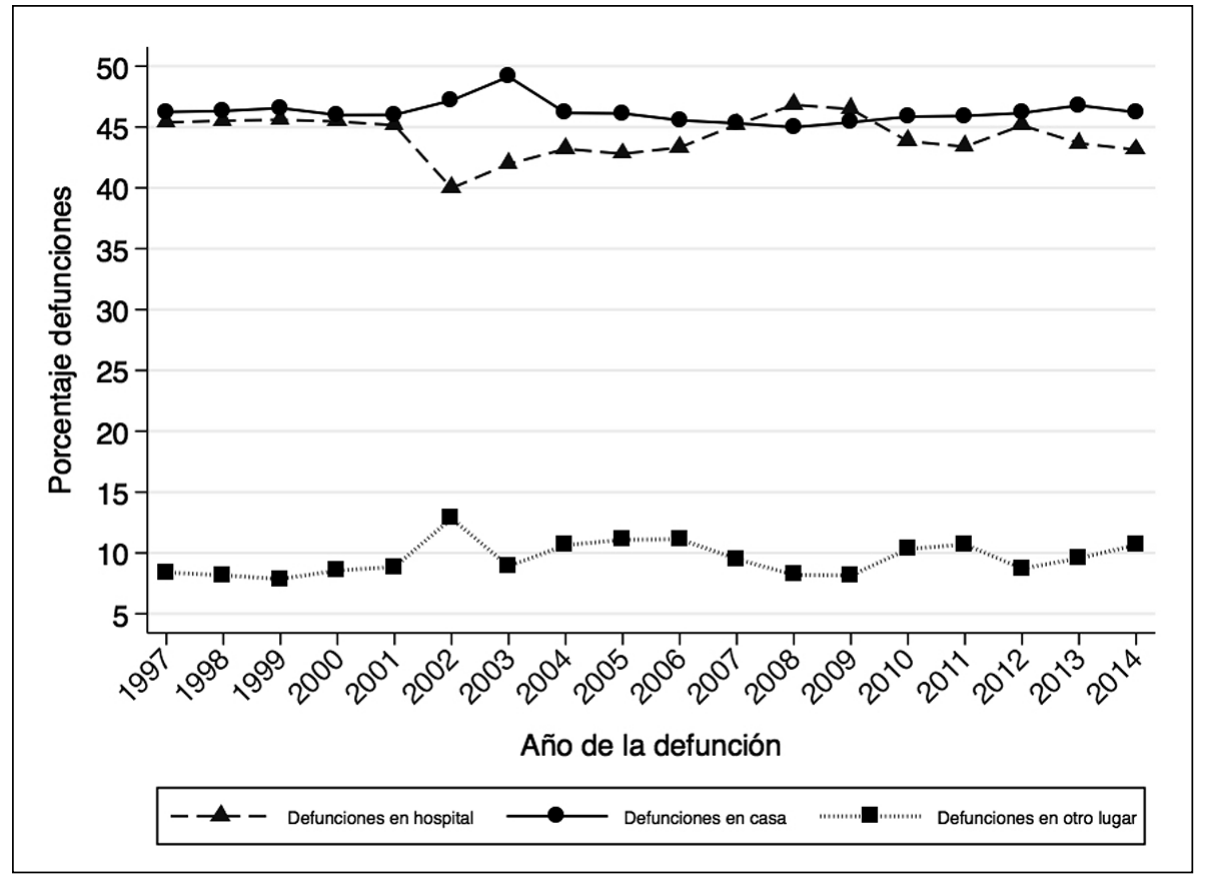

Figura 3. Tendencia de defunciones según lugar de ocurrencia, Chile 1997-2014. 


\section{Discusión}

El presente estudio evidenció que la mayoría de las defunciones durante el período estudiado ocurrió en la casa o habitación (46\%) y menos de $10 \%$ en otro lugar. El análisis temporal mostró que no hubo variaciones en cuanto al lugar de ocurrencia de la muerte durante el período de estudio. Los factores asociados a muerte en domicilio fueron sexo femenino, ruralidad, estado civil casado o viudo y menor nivel educacional para los menores de 70 años, en cambio en los de 70 o más años, tanto el estado civil casado, como el mayor nivel educacional, se asociaron a muerte en el hospital.

En cuanto a la distribución del lugar de ocurrencia de la defunción, el estudio de Broad et al., que incluyó Chile, encontró cifras similares, reportando que entre los años 2001 y 2010,47\% de las defunciones ocurre en hospital y $53 \%$ en casa u otro lugar; sin embargo, este estudio no evaluó características sociodemográficas asociadas al lugar de defunción, ya que el objetivo fue determinar el lugar de la muerte 9 . En relación a la tendencia, no se encontraron estudios similares en Chile que estratifiquen por lugar de defunción; sin embargo, los resultados coinciden con lo encontrado en Estados Unidos de Norteamérica en el año 2013 por Hall et al., que muestra que entre los años 2000 y 2010 el porcentaje de muertes ocurridas en el hospital no experimentó cambios significativos ${ }^{13}$.

El resultado más destacable es que, al estratificar la edad por decenios, se observa que hasta el grupo de 60 a 69 años las defunciones se producen mayoritariamente en contextos hospitalarios; el cambio a defunción en la casa ocurre desde los 70 años en adelante. Esto es coherente con la evidencia nacional ${ }^{10}$ e internacional que demuestra que a mayor edad existe una inclinación a morir en lugares fuera del hospital ${ }^{14,15}$. Específicamente, las probabilidades de morir en el hogar aumentan gradualmente con la edad en países como Francia, Italia, España, México y Corea $^{16}$. Para mayores de 65 años, la menor proporción de fallecidos en hospital se presenta en Estados Unidos de Norteamérica y los Países Bajos, mientras que en Bélgica, Inglaterra, Alemania y Noruega la mayor proporción lo hace en hospital ${ }^{17}$.

En relación a las variables sociodemográficas, los hombres mueren en mayor proporción en el hospital, mientras que las mujeres lo hacen en casa, situación similar a lo reportado en otros estudios $^{18,19}$. Las personas casadas o viudas tienen mayor probabilidad de morir en la casa en los menores de 70 años. Una explicación al respecto es que en las personas de menor edad, la mayoría de las defunciones ocurre por causas externas o agudas $^{20,21}$. Por otro lado, en el grupo de mayores de 70 años, solo los viudos tienden a fallecer más en la casa, mientras que los casados lo hacen el hospital. En este grupo, las muertes se deben principalmente a enfermedades crónicas no transmisibles, por lo tanto, estar casado podría favorecer el acceso a atención hospitalaria ${ }^{20}$.

En relación al nivel educacional, en los menores de 70 años, a mayor nivel educacional, hay una mayor probabilidad de fallecer en hogar, a diferencia de los mayores de 70, en los cuales todos aquellos con algún tipo de nivel educacional tienen menor probabilidad de fallecer en el hogar, en comparación a aquellos con ningún nivel educacional. En la literatura internacional no se encuentran resultados consistentes, lo cual podría estar explicado por confusión residual ${ }^{22}$, esto es, que el efecto pueda estar distorsionado por la no medición de otras variables.

La mayoría de las defunciones en Chile se produjo en personas mayores que se encontraban inactivas laboralmente. El área rural se asoció a una mayor probabilidad de fallecer en casa o habitación, lo cual podría explicarse por las dificultades de acceso y menor disponibilidad de atención médica ${ }^{23}$.

En relación a las fortalezas, este estudio se destaca por su representatividad, al haber incluido 18 años de defunciones ocurridas en Chile, lo que evaluó la tendencia en base a datos poblacionales. Además, se presenta el primer estudio que analiza la relación entre el lugar de la defunción y características sociodemográficas en Chile. Estos resultados complementan el estudio de Leiva et al., que describió el lugar de la defunción en un período de 13 años en Chile, según edad ${ }^{10}$.

Entre las limitaciones cabe mencionar el uso de bases de datos secundarias, las cuales no son recolectadas con fines de investigación, sino administrativos, por lo cual no contiene necesariamente toda la información necesaria. En este estudio es importante considerar ciertas variables con precaución, por ejemplo, el estado civil, ya que la forma de codificar la variable impide el reconocimiento de personas que conviven y que, 
por lo tanto, al igual que los casados, cuentan con una red de apoyo inmediata.

De los resultados se desprende que las defunciones domiciliarias aumentan en la medida que aumenta la edad, lo que se evidenció por el aumento de las defunciones en casa al evaluar por decenios de edad. Se identifican otros factores sociodemográficos que condicionan el lugar en la muerte, que posiblemente se relacionan con una pobre red social y problemas de acceso a la atención hospitalaria.

Sin embargo, también debe considerarse que la situación de fallecer en el hospital puede no ser deseada; estudios han evidenciado que la defunción en el hospital se caracteriza por falta de intimidad, recursos y apoyo emocional ${ }^{24}$. Por otro lado, la defunción en domicilio otorga condiciones de dignidad y acompañamiento para el difunto y la familia, siendo, además, de preferencia para las personas ${ }^{25,26}$.

Los resultados de la presente investigación servirán de insumo para la discusión sobre los recursos y políticas públicas, no solo en términos de acceso a atención de salud hospitalaria, sino también a cobertura y enfoque de la muerte en domicilio, considerando que la evidencia internacional $^{27-30}$ establece la preferencia de las personas por vivir este proceso en su hogar, cuando la muerte se puede prever.

\section{Referencias}

1. Chile, Ministerio de Salud. Esperanza de vida al nacer (en años), según sexo, por quinquenios comprendidos entre los años 1950 y 2025. Santiago, Chile: Autor, Departamento de Estadística e Información en Salud. 2004.

2. Celis Rodríguez E, Rubiano S. Desarrollo del cuidado intensivo en Latinoamérica. Todo Hosp 2007; 234: 97-100. Disponible en: http://pesquisa.bvs.br/brasil/resource/pt/ ibc-61872.

3. Chile, Instituto Nacional de Estadísticas. Adulto mayor en Chile. Santiago, Chile: Autor; 2007. Disponible en www.ine.cl/docs/default-source/FAQ/enfoque- estadístico-adulto-mayor-en-chile.pdf?sfvrsn=2.

4. Organización Mundial de la Salud. Envejecimiento y ciclo de vida. Washington, DC: Autor. Disponible en: http://www.who.int/features/factfiles/ageing/ageing_facts/es/.

5. Chile, Instituto Nacional de Estadísticas, Comisión Económica para América Latina y el Caribe. Chile: proyecciones y estimaciones de población. Total país: 1950-
2050. Santiago, Chile: Autores, División de Población, Centro Latinoamericano y Caribeno de Demografía; 2005. Disponible en: http://historico.ine.cl/canales/ chile_estadistico/demografia_y_vitales/proyecciones/ Informes/MicrosoftWordInforP_T.pdf

6. Ariès P. Historia de la muerte en occidente: desde la edad media hasta nuestros días (Carbajo F, Perrin R, Trads.; Título original: Essais sur l'histoire de la mort en occident : du moyen âge à nos jours). Barcelona, España: El Acantilado; 1975/2000.

7. Benavente Aninat MA. La concepción de la muerte y el funeral en Chile. Rev Chil Antropol 2005-2006; 18: 93104.

8. Rovaletti ML. La ambigüedad de la muerte: reflexiones en torno a la muerte contemporánea. Rev Colomb Psiquiatr 2002; 31 (2): 137-54.

9. Broad JB, Gott M, Kim H, Boyd M, Chen H, Connolly MJ. Where do people die? An international comparison of the percentage of deaths occurring in hospital and residential aged care settings in 45 populations, using published and available statistics. Int J Public Health 2013; 58 (2): 257-67.

10. Leiva H, León KF. Cobertura de la atención de la enfermedad que causa la muerte y lugar de ocurrencia del deceso, en Chile y la sexta región, 1990-2003. Rev Med Chile 2007; 135 (8): 1025-33.

11. Núñez F ML, Icaza N MG. Calidad de las estadísticas de mortalidad en Chile, 1997-2003. Rev Med Chile 2006; 134 (9): 1191-6.

12. Cavada CG. Regresión de Prais-Winsten: evaluación de tendencias lineales. Rev Chil Endocrinol Diabetes 2015; 8 (4): 176-7.

13. Hall MJ, Levant S, DeFrances CJ. Trends in inpatient hospital deaths: National Hospital Discharge Survey, 2000-2010. NCHS Data Briefs 2013; 118: 1-8.

14. Evans CJ, Ho Y, Daveson BA, Hall S, Higginson IJ, Gao W. Place and cause of death in centenarians: A population-based observational study in England, 2001 to 2010. PLoS Med 2014; June: e1001653. Disponible en: http://dx.plos.org/10.1371/journal.pmed.1001653.

15. Sleeman KE, Ho YK, Verne J, Gao W, Higginson IJ. Reversal of English trend towards hospital death in dementia: A population-based study of place of death and associated individual and regional factors, 2001-2010. BMC Neurol 2014; 14 (1): 59.

16. Hedinger D, Braun J, Zellweger U, Kaplan V, Bopp M, for the Swiss National Cohort Study Group. Moving to and dying in a nursing home depends not only on health - An analysis of socio-demographic determinants of place of death in Switzerland. PLoS One 2014; 9 (11): e113236. 
17. Bekelman JE, Halpern SD, Blankart CR, Bynum JP, Cohen J, Fowler R, et al. Comparison of site of death, health care utilization, and hospital expenditures for patients dying with cancer in 7 developed countries. JAMA 2016; 315 (3): 272-83.

18. Reyniers T, Deliens L, Pasman HR, Morin L, Addington-Hall J, Frova L, et al. International variation in place of death of older people who died from dementia in 14 European and non-European countries. J Am Med Dir Assoc 2015; 16 (2): 165-71.

19. Houttekier D, Cohen J, Bilsen J, Deboosere P, Verduyckt $\mathrm{P}$, Deliens L. Determinants of the place of death in the Brussels Metropolitan Region. J Pain Symptom Manage 2009; 37 (6): 996-1005.

20. Chile, Ministerio de Salud. Defunciones y mortalidad general y por grupos de edad. Santiago, Chile: Autor, Departamento de Estadísticas e Información de Salud; 2011-2014. Disponible en: http://www.deis.cl/defunciones-y-mortalidad-general-y-por-grupos-de-edad/.

21. U.S. Department of Health \& Human Services Centers for Disease, Control and Prevention. 10 Leading causes of death by age group, United States - 2015. Washington, DC: Autor, National Center for Injury Prevention and Control; 2015. Disponible en: https://www.cdc.gov/ injury/wisqars/pdf/leading_causes_of_death_by_age_ group_2015-a.pdf.

22. Gisquet E, Julliard S, Geoffroy-Pérez B. Do social factors affect the place of death? Analysis of home versus institutional death over 20 years. J Public Health 2016; 38 (4): e472-9.

23. Fábrega R. Salud y desigualdad territorial (Documentos de Trabajo $\mathrm{N}^{\circ}$ 13). Santiago, Chile: Centro Latinoamericano para el Desarrollo Rural; 2013. Disponible en: http://www.rimisp.org/wp/-content/files_mf/
1375105909Doc13SaludCTDFABREGA.pdf.

24. Ruiz-Fernández MD, Ortega-Galán AM, Cabrera-Troya J, Ibáñez-Masero O, Carmona-Rega MI, Relinque-Medina $\mathrm{F}$, et al. Personas enfermas al final de la vida: vivencias en la accesibilidad a recursos sociosanitarios. Enfermería Universitaria 2017; 14 (1): 108.

25. Wilson DM, Cohen J, Deliens L, Hewitt JA, Houttekier D. The preferred place of last days: Results of a representative population-based public survey. J Palliat Med 2013; 16 (5): 502-8.

26. Higginson IJ, Sarmento VP, Calanzani N, Benalia H, Gomes B. Dying at home-is it better: A narrative appraisal of the state of the science. J Palliat Med 2013; 27 (10): 918-24.

27. Gomes B, Higginson IJ. Factors influencing death at home in terminally ill patients with cancer: systematic review. BMJ 2006; 332 (7540): 515-21.

28. Londoño JL, Frenk J. Pluralismo estructurado: hacia un modelo innovador para la reforma de los sistemas de salud en América Latina. Washington, DC: Inter-American Development Bank; 1997. Disponible en: https://www.econstor.eu/bitstream /10419/87917/1/ idb-wp_353.pdf.

29. Ganga Contreras F, Piñones Santana MA, González Vásquez D, Rebagliati Badal F. Rol del Estado frente al envejecimiento de la población: el caso de Chile. Convergencia 2016; 23 (71): 175-99. Disponible en: http:// www.scielo.org.mx/scielo.php?script=sci_arttext\&pi$\mathrm{d}=$ S1405-14352016000200175.

30. México, Secretaría de Salud. Muerte digna: una oportunidad real. México, DF: Autor, Comisión Nacional de Bioética; 2008. Disponible en: http://www.conbioetica-mexico.salud.gob.mx/descargas/pdf/publicaciones/ memorias/muertedigna.pdf. 